\title{
Drug Delivery Formulations and Devices Tailored for Paediatric and Older Patients
}

\author{
David J. Brayden * \\ University College Dublin (UCD) School of Veterinary Medicine, UCD Conway Institute of Biotechnology, CÚRAM, the Science \\ Foundation Ireland Research Centre for Medical Devices, UCD, Belfield, Dublin, Ireland
}

Keywords: paediatric drug delivery, delivery in older persons, compliance, efficacy, adapted delivery for special populations, palatibility, excipients, dysphagia

"Drugs don't work in patients who don't take them," C. Everett Coop, US Surgeon General (1985). This is a quote widely used in discussions of poor compliance with drug dosing regimens. It is particularly apt in relation to the non-adherence to medications by children and older persons, where ascertaining correct doses in the context of physiological differences according to age profiles is a key consideration in making acceptable formulations and devices for delivery. The concept of the average patient is a myth-there is no average child or older person, hence one formulation will not necessarily be safe and effective for all patients. When a new medicine is submitted to regulatory agencies for approval, clinical trials are generally carried out on patients over the age of 18 and under the age of 65. Trials in "average" patients aim to determine if a medicine will be safe and efficacious, but drugs are also required for patients that lie outside of the average, including paediatrics and older persons. Few drugs have undergone clinical trials in paediatric patients, so in the absence of specific formulations, physicians can opt to use such drugs off-label and/or in an unlicensed fashion (Chen et al., 2021). Off-label use is one when a licenced product is used outside the specifics of the license or label granted by the national regulator. For use in paediatrics and older persons, this might include the age range, the dose level, or its use in other clinical indications.

A common mantra in the field of paediatrics is that "children are not small adults". Childhood spans pre-term infants, full term infants ( $0-28$ days), infants and toddlers ( 28 days- 23 months), children from 2-11 years old, and adolescents from 12 to 16/18 years old. In the absence of a licenced drug, physicians tend to adjust the dose of a drug licenced in adults on a $\mathrm{mg} / \mathrm{kg}$ basis, use a lower dose strength, or opt for a compounded formulation where preparation errors can be made. In many cases there will be no difference between how a dosage form performs in adults and children and safety and efficacy will not be compromised. The differences between adults and children relevant to dosing include weight, height, body water content, metabolism, gastric $\mathrm{pH}$, gastric emptying, sensitivity to drugs and excipients, and hepatic clearance. Pharmacokinetic (PK) changes may arise in paediatric patients due to differences in body water content that can influence distribution. There may also be altered expression of transporters, enzymes, and carriers that in turn impact absorption, distribution, metabolism, and excretion. Altered expression of metabolising enzymes in paediatric patients can have a profound effect on PK parameters leading to compromised drug and excipient safety. Similarly, the expression of drug targets may be reduced, and this may potentially affect the pharmacodynamic (PD) outcome.

When it comes to the formulation, manipulating a dosage form to aide swallowing or to reduce the dose in paediatrics can lead to product instability and lack of bioequivalence with the adult formulation, which adds further to the unpredictable drug behaviour in this patient population. In recent years the European Medicines Agency (2017) has drafted Guidances to promote paediatric medicine development and to create clearer pathways for the paediatric clinical testing of therapeutics

David david.brayden

Received: 21 October 2021 Accepted: 15 November 2021

Citation:

Brayden DJ (2021) Drug Delivery Formulations and Devices Tailored for Paediatric and Older Patients. Front. Drug. Deliv. 1:782635. doi: 10.3389/fddev.2021.782635
(EMA, 2020). It also requires applicants to agree a Paediatric Implementation Plan (PIP) with it if 
seeking authorisation of a medicine for children (https://www. ema.europa.eu/en/human-regulatory/research-development/ paediatric-medicines/paediatric-investigation-plans ). The US Food and Drugs Administration (2020) has also recently updated its draft guidance on developing paediatric medicines for rare diseases (US FDA, 2017). Applicants are subject to the US Pediatric Research Equity Act and the Best Pharmaceuticals for Children Act, and must provide a Pediatric Study Plan. There is ongoing effort to reduce the regulatory barriers for this branch of pharmaceutical science. The principle advocated by regulatory agencies is that we should be administering therapeutics to paediatric patients following testing in that population (Karish, 2015). Pharma will usually only engage if there is a potential market or market exclusivity incentives, the latter now being offered by EMA and the FDA. Clinical trials are especially hard to do in paediatric populations and are associated with higher risk due to issues over numbers of participants, compliance, and ethics. This will take investment, but stakeholders will need to be convinced that there is a real problem for more than a handful of drugs being used in children. At a minimum, research could be promoted in paediatric drug development in respect of compiling an excipient database, a compounding framework, and investment in compounding facilities with an emphasis on stability and product shelf-life studies. There is also scope for adapting adult dosage forms that can be manipulated to assist swallowing in paediatric patients and to enable dose reductions.

In 2015, Innovate United Kingdom co-funded an open innovation consortium (SPaeDD-UK: Smart Paediatric Drug Development-United Kingdom; http://www. paediatricscienceuk.com/paediatric-medicines.html) of academic groups and industry partners to provide appropriate tools for palatability testing, taste masking solutions, biorelevant paediatric GI dissolution media, as well as delivery platforms. It was supported by many of the largest United Kingdom Pharma companies, each with formulation expertise. An important review also called for more evidence to guide formulation development for neonates and paediatrics (Batchelor and Marriott, 2013). They also emphasised the importance of using the lowest effective dose, palatability and taste masking, bioavailability, convenient administration routes and devices, the use of appropriate excipients, along with more cost-effective production. Even if a paediatric formulation could be made as a perfectly scaled version of the adult one, it does not solve the problem of altered performance of the drug if its $\mathrm{PK}$ is influenced by physiology and anatomical developmental changes, or if drug target expression is different (Ivanovska et al., 2014).

On the drug delivery side, Vinarov et al. (2021) and Stillhart et al. (2020) from the EU COST Action Project, The European Network on Understanding Gastrointestinal Absorption-related Processes (UNGAP), have highlighted that the study of oral formulations and drug absorption processes in neonates and paediatrics is still at an early stage and that GI diseases and conditions that indirectly effect the GI tract can also influence absorption. They advocate development of more accurate in vitro biorelevant dissolution and modelling to represent oral dosage form performance in these populations. Such methodology is needed to better predict outcomes from oral dosage forms designed for the young including liquids and solids (Thabet et al., 2018). Recent work examined dissolution of two small molecules in simulated paediatric intestinal fluids containing infant formulae an attempt to gain further in-depth understanding of food effects on oral dosage forms in paediatric patients (Kofoed-Djursner et al., 2021; Vinarov et al., 2021). Virtually every route of delivery requires an adapted use of formulations and devices in neonates (Linakis et al., 2016) and paediatrics including parenteral through to nasal (Swedrowska et al., 2021), pulmonary (Arzu, 2021), oromucosal (Dahmash et al., 2021), and transdermal (Duarah et al., 2019). Recent examples of such efforts were the use of adapted nasogastric tubes to deliver crushed tablets to paediatric patients (Swedrowska et al., 2021) and an orodispersable tablet to improve patient adherence by avoiding the requirement to swallow a solid dosage form (Wagner-Hattler et al., 2021). There is therefore much to be done to better understand physiology in paediatric patients and to design formulations that allow accurate and convenient administration (Mfoafo et al., 2021). Strickley (2019) has predicted that oral formulations for paediatrics will be dominated by multi-particulates comprising oral disintegrating tablets, sprinkle capsules, and minitablets in simple packaging presentations.

Excipient research also needs to be encouraged to replace potentially harmful examples in paediatric dosage forms to safely maximise performance, stability, and palatability (Saito et al., 2021). Examples of potentially harmful excipients in neonates and infants include ethanol (neurotoxicity), benzyl alcohol (neurotoxicity, metabolic acidosis), the polysorbates (liver and kidney failure), and propylene glycol (seizures, hyperosmolarity, metabolic acidosis, and neurotoxicity) (Rouaz et al., 2021). Salunke et al. (2021) described a recent stakeholder workshop to discuss new approaches for excipient selection for age-appropriate paediatric formulations with input from Pharma and regulators. There is only a limited selection of excipients allowed for paediatric oral formulations, maximum allowable concentrations for some (5\% for ethanol), and issues around carbohydrate concentrations and diet. Finally, delivery challenges for paediatrics was the subject of a recent special conference of the Controlled Release Society (https:// www.controlledreleasesociety.org/crs-2021-pediatric-drugdevelopment-delivery-meeting), an indication that it has become a centre-stage topic.

Medication use in old age covers a far longer period than paediatrics, several decades. Older persons can be grouped into two age ranges; "young" elderly (70-85 years) and the "old" elderly ( $>85$ years), but it is unwise to define a patient purely by age. As is the case with paediatrics, there are changes in old persons including changes in the oral cavity (xerostomia), delayed oesophageal transit, dysphagia, decreased gastric acid production, reduced gastric emptying time, reduced intestinal surface area, declining renal and hepatic function, as well as differences in cognition, visual acuity, and dexterity. Although medicines developed in pre-age 65 adults are widely used in older people, the latter tend to be either excluded or are underrepresented in clinical trials (Barry and Hughes, 2021). This is because older subjects have co-morbidities and have higher risks of drug interactions, many of which are only discovered through 
the reporting of adverse drug reactions during pharmacovigilance. Most patients over the age of 65 are taking more than five drugs concomitantly for at least three conditions, so there is enormous potential for PK interference in absorption and metabolism, especially when using low therapeutic index molecules. The FDA has issued an updated draft guidance on correct labelling to promote the safe and effective use of medicines in older persons (FDA, 2020). They note that use of a product in older patients may not be justified if insufficient numbers of such patients were included in the original clinical trials. The EMA has also produced reflections on the topic, highlighting the benefits of adapted formulations for such patients (EMA, 2017). A recent review has assessed organ changes in sight, hearing, swallow capacity, as well as motor and cognitive function in subjects over the age of 75 and concluded that these changes hugely impact adherence to dosing regimens and the correct use of drug-device combinations-all of which can combine to reduce safety and efficacy (Drenth-van Maanen et al., 2020). Others are attempting for the first time to assess the influence of chronic disease in the elderly on gastric retention time, gastric transit, composition of gastrointestinal fluids, absorption processes in the intestine and on the fate and performance of oral dosage forms (Vinarov et al., 2021; Stillhart et al., 2020).

For low therapeutic index drugs in particular, formulations and delivery systems can be specially designed for paediatrics and older patients, and some systems may have some features in common. For oral delivery, standard capsules and tablets may be replaced by lozenges, oromucosal films, sub-lingual capsules, taste-masked effervescent drinks, and oral formulations based on sprinkled granules or chewable systems. The feasibility of creating such formulations will ultimately depend on the physicochemical properties of the drug, potency, efficacy, and safety considerations. These can address issues around inefficient swallowing and reduced saliva production. However, any specially designed formulation where the dissolved form of the drug is presented in the oral cavity for ease of swallowing will require attention to taste masking for older subjects. For inhaled delivery, where such patients may have difficulty breathing due to conditions such as chronic pulmonary fibrosis, standard systems can in theory be replaced by ageappropriate actuated metered systems and nebulisers (Wallin et al., 2018). Yet, there are few pulmonary delivery systems that have been specifically developed for older people despite the

\section{REFERENCES}

Ari, A. (2021). A Path to Successful Patient Outcomes through Aerosol Drug Delivery to Children: a Narrative Review. Ann. Transl. Med. 9 (7), 593. doi:10.21037/atm-20-1682

Barry, H. E., and Hughes, C. M. (2021). An Update on Medication Use in Older Adults: a Narrative Review. Curr. Epidemiol. Rep. 8, 108-115. doi:10.1007/ s40471-021-00274-5

Batchelor, H. K., and Marriott, J. F. (2015). Formulations for Children: Problems and Solutions. Br. J. Clin. Pharmacol. 79 (3), 405-418. doi:10.1111/bcp.1226 unmet medical need. Injections can also be modified for formulations suited to high gauge needles and in longacting formats, while implants can be altered in drug loading, design, and composition for elderly. Transdermal patches need to be designed for older populations with different loadings of certain active agents to cater for differences in skin thickness and permeability from the main adult population (Kaestli et al., 2008). Additive manufacturing for formulation and devices and 3-D printing can potentially be used to design delivery systems adapted for the needs of special populations including the young and older persons (Karavasili et al., 2021). Overall, specialised delivery systems for these populations are currently based on niche products where there is market demand rather than being part of the mainstream of drug delivery formulation and device research. Innovation to improve drug delivery systems for the elderly is therefore being advocated to address the problems of polypharmacy, poor adherence to dosing, co-morbidities, and substantial variations in PK and PD (Khan and Roberts, 2018).

At Frontiers in Drug Delivery, we are interested in promoting research into how formulations and delivery systems impact these unique populations in respect of drug administration and performance. Physiological differences from the typical adult population can account for major difference in the outcomes. Effort is now needed to support specific research projects in comparative physiology, drug formulation, and devices to make well-designed efficacious and safe delivery systems for paediatric and older patients. It is time to support more drug delivery research effort for these cohorts representing millions of patients who are currently poorly served. It is indeed an ambitious and truly grand challenge for our journal.

\section{AUTHOR CONTRIBUTIONS}

DB wrote the article in its entirety.

\section{ACKNOWLEDGMENTS}

I thank Dr. Sam Maher of the School of Pharmacy and Biomolecular Sciences at the Royal College of Surgeons in Ireland for helpful discussions.

Chen, Z., Li, S., Zeng, L., Liu, Y., Zhang, M., Choonara, I., et al. (2021). Accessibility of Medicines for Children: a Systematic Review. Front. Pharmacol. 12, 691606 doi:10.3389/fphar.2021.691606

Dahmash, E. Z., Iyire, A., and Alyami, H. S. (2021). Development of Orally Dissolving Films for Pediatric-Centric Administration of Anti-epileptic Drug Topiramate - A Design of Experiments (DoE) Study. Saudi Pharm. J. 29 (7), 635-647. doi:10.1016/j.jsps.2021.04.025

Drenth-van Maanen, A. C., Wilting, I., and Jansen, P. A. F. (2020). Prescribing Medicines to Older People-How to Consider the Impact of Ageing on Human Organ and Body Functions. Br. J. Clin. Pharmacol. 86 (10), 1921-1930. doi:10.1111/bcp.14094 
Duarah, S., Sharma, M., and Wen, J. (2019). Recent Advances in MicroneedleBased Drug Delivery: Special Emphasis on its Use in Paediatric Population. Eur. J. Pharmaceutics Biopharmaceutics 136, 48-69. doi:10.1016/j.ejpb.2019.01.005

European Medicines Agency (2020). European Medicines Agency and European Commission (DG Health and Food Safety) Action Plan on Paediatrics. Available at: https://www.ema.europa.eu/en/documents/report/europeanmedicines-agency-european-commission-dg-health-food-safety-action-planpaediatrics_en.pdf (Accessed September $\left.1^{\text {st }}, 2021\right)$.

European Medicines Agency (2017). Reflection Paper on the Pharmaceutical Development of Medicines for Use in the Older Population. EMA/CHMP/ QWP/292439/2017. Available at: https://www.ema.europa.eu/en/documents/ scientific-guideline/reflection-paper-pharmaceutical-development-medicinesuse-older-population-first-version_en.pdf (Accessed September 1st, 2021).

Food and Drugs Administration (2020). Geriatric Information in Human Prescription Drug and Biological Product Labeling: (Draft) Guidance for Industry. Available at: https://www.fda.gov/media/142162/download (Accessed September 1st, 2021).

Food and Drugs Administration (2017). Pediatric Rare Diseases-A Collaborative Approach for Drug Development Using Gaucher Disease as a Model; Draft Guidance for Industry. Available at: https://www.fda.gov/regulatory-information/ search-fda-guidance-documents/pediatric-rare-diseases-collaborative-approachdrug-development-using-gaucher-disease-model-draft (Accessed September 1st, 2021).

Ivanovska, V., Rademaker, C. M. A., van Dijk, L., and Mantel-Teeuwisse, A. K. (2014). Pediatric Drug Formulations: a Review of Challenges and Progress. Pediatrics 134 (2), 361-372. doi:10.1542/peds.2013-3225

Kaestli, L.-Z., Wasilewski-Rasca, A.-F., Bonnabry, P., and Vogt-Ferrier, N. (2008). Use of Transdermal Drug Formulations in the Elderly. Drugs \& Aging 25 (4), 269-280. doi:10.2165/00002512-200825040-00001

Karavasili, C., Eleftheriadis, G. K., Gioumouxouzis, C., Andriotis, E. G., and Fatouros, D. G. (2021). Mucosal Drug Delivery and 3D Printing Technologies: A Focus on Special Patient Populations. Adv. Drug Deliv. Rev. 176, 113858. doi:10.1016/j.addr.2021.113858

Karish, A. (2015). Available at: https://www.fda.gov/files/drugs/published/ Pediatric-Drug-Development-Regulatory-Expectations.pdf (Accessed September $\left.1^{\text {st }}, 2015\right)$.

Khan, M. S., and Roberts, M. S. (2018). Challenges and Innovations of Drug Delivery in Older Age. Adv. Drug Deliv. Rev. 135, 3-38. doi:10.1016/ j.addr.2018.09.003

Kofoed-Djursner, C., Jamil, A., Selen, A., Müllertz, A., and Berthelsen, R. (2021). Drug Solubilization during Simulated Pediatric Gastro-Intestinal Digestion. Eur. J. Pharm. Sci. 162, 105828. doi:10.1016/j.ejps.2021.105828

Linakis, M. W., Roberts, J. K., Lala, A. C., Spigarelli, M. G., Medlicott, N. J., Reith, D. M., et al. (2016). Challenges Associated with Route of Administration in Neonatal Drug Delivery. Clin. Pharmacokinet. 55 (2), 185-196. doi:10.1007/ s40262-015-0313-z

Mfoafo, K. A., Omidian, M., Bertol, C. D., Omidi, Y., and Omidian, H. (2021). Neonatal and Pediatric Oral Drug Delivery: Hopes and Hurdles. Int. J. Pharmaceutics 597, 120296. doi:10.1016/j.ijpharm.2021.120296

Rouaz, K., Chiclana-Rodríguez, B., Nardi-Ricart, A., Suñé-Pou, M., MercadéFrutos, D., Suñé-Negre, J. M., et al. (2021). Excipients in the Paediatric
Population: a Review. Pharmaceutics 13 (3), 387. doi:10.3390/ pharmaceutics 13030387

Saito, J., Nadatani, N., Setoguchi, M., Nakao, M., Kimura, H., Sameshima, M., et al. (2021). Potentially Harmful Excipients in Neonatal Medications: a Multicenter Nationwide Observational Study in Japan. J. Pharm. Health Care Sci. 7 (1), 23. doi:10.1186/s40780-021-00208-9

Salunke, S., Clapham, D., Agrawal, A., Hughes, K., and Nunn, T. (2021). Best Practices for Selection of Excipients for Paediatrics - Workshop Reflection. Eur. J. Pharmaceutics Biopharmaceutics 160, 77-81. doi:10.1016/ j.ejpb.2020.12.021

Stillhart, C., Vučićević, K., Augustijns, P., Basit, A. W., Batchelor, H., Flanagan, T. R., et al. (2020). Impact of Gastrointestinal Physiology on Drug Absorption in Special Populations--An UNGAP Review. Eur. J. Pharm. Sci. 147, 105280. doi:10.1016/j.ejps.2020.105280

Strickley, R. G. (2019). Pediatric Oral Formulations: An Updated Review of Commercially Available Pediatric Oral Formulations since 2007. J. Pharm. Sci. 108 (4), 1335-1365. doi:10.1016/j.xphs.2018.11.013

Swedrowska, M., Ingham, S., Tomlin, S., and Forbes, B. (2021). Recommendations for Crushing Circadin (Melatonin) Tablets for Safe and Reliable Delivery via Pediatric Nasogastric Tubes. Int. J. Pharmaceutics 594, 120151. doi:10.1016/ j.ijpharm.2020.120151

Thabet, Y., Klingmann, V., and Breitkreutz, J. (2018). Drug Formulations: Standards and Novel Strategies for Drug Administration in Pediatrics. J. Clin. Pharmacol. 58 (Suppl. 10), S26-S35. doi:10.1002/jcph.1138

Vinarov, Z., Abrahamsson, B., Artursson, P., Batchelor, H., Berben, P., BernkopSchnürch, A., et al. (2021). Current Challenges and Future Perspectives in Oral Absorption Research: An Opinion of the UNGAP Network. Adv. Drug Deliv. Rev. 171, 289-331. doi:10.1016/j.addr.2021.02.001

Wagner-Hattler, L., Kiene, K., Bielicki, J., Pfister, M., Puchkov, M., and Huwyler, J. (2021). High Acceptability of an Orally Dispersible Tablet Formulation by Children. Children (Basel). 8 (3), 194. doi:10.3390/children8030194

Wallin, M., Tagami, T., Chen, L., Yang, M., and Chan, H.-K. (2018). Pulmonary Drug Delivery to Older People. Adv. Drug Deliv. Rev. 135, 50-61. doi:10.1016/ j.addr.2017.11.010

Conflict of Interest: The author declares that the research was conducted in the absence of any commercial or financial relationships that could be construed as a potential conflict of interest.

Publisher's Note: All claims expressed in this article are solely those of the authors and do not necessarily represent those of their affiliated organizations, or those of the publisher, the editors and the reviewers. Any product that may be evaluated in this article, or claim that may be made by its manufacturer, is not guaranteed or endorsed by the publisher.

Copyright $\odot 2021$ Brayden. This is an open-access article distributed under the terms of the Creative Commons Attribution License (CC BY). The use, distribution or reproduction in other forums is permitted, provided the original author(s) and the copyright owner(s) are credited and that the original publication in this journal is cited, in accordance with accepted academic practice. No use, distribution or reproduction is permitted which does not comply with these terms. 